\title{
Study the Effect of Solvent / Non-solvent on Polycarbonate
}

\author{
Raad S. Sabry, Muntazer I. Al-mosawi* \\ Physics Department, College of Science, Al-Mustansiriyah University, Baghdad, Iraq
}

Email address:

superhydrophobicsurfaces@mail.ru (M. I. Al-mosawi)

${ }^{*}$ Corresponding author

To cite this article:

Raad S. Sabry, Muntazer I. Al-mosawi. Study the Effect of Solvent / Non-solvent on Polycarbonate. American Journal of Nanosciences. Vol. 3, No. 3, 2017, pp. 59-62. doi: 10.11648/j.ajn.20170303.15

Received: March 20, 2017; Accepted: April 1, 2017; Published: May 3, 2017

\begin{abstract}
A stable superhydrophobic PC surface was obtained by a simple phase separation method at room temperature. The water contact angle of the superhydrophobic PC surface is $156 \pm 2^{\circ}$. FESEM image exhibited that the PC surface composed of flower-like shaped linked together centrally with branches offered roughness on the surface with a hierarchical micro/nano-binary formation. When the PC superhydrophobic surface was immersed in water with the temperatures ranging from $5^{\circ} \mathrm{C}$ to $45^{\circ} \mathrm{C}$ for $1 \mathrm{~h}$ to 15 days, the water contact angle remained higher than $150^{\circ}$.
\end{abstract}

Keywords: Superhydrophobic Surfaces, Biomimetic Surfaces, Phase Separation, Solvent / Non-solvent, Acetone, DMF, Polycarbonate, Self-Cleaning

\section{Introduction}

Biomimetic surfaces have the greatest attention of engineers and scientists due to their unusual properties, biological structures can be mimetic in many types of surfaces such as Lotus leaves, Mosquito-moth eyes, Butterfly wings, Rice leaves, Cicada wings, Gecko feet, Desert beetle, Spider silks, fish scales which exhibit excellent hydrophobic $[1,2]$. These natural structures show new idea in design of superhydrophobic structures, superhydrophobic surfaces, defined as a surface that a drop of water almost perfect sphere and even a very slight tilting cause the water drop to roll off, and have a high water contact angle (CA) and low sliding angle, the ability of a surface to water-repellency droplets that consider important for both biological and technical applications [3]. In nature, lotus leaves exhibit specific properties of superhydrophobicity and self-cleaning which considered one of the primary surface properties of solid materials usually characterized by measurement of the contact angle of a surface [4]. Generally, surfaces with water contact angles larger than $150^{\circ}$ and slide angles lower than $10^{\circ}$ can easily rolling the water drops and called superhydrophobic surfaces $[5,6]$. Nowadays, most of researchers focused on superhydrophobic surfaces due to unique applications used in many fields, such as selfcleaning, oil-water separation, anti-reflection, anti-adhesive, fluidic drag reduction, anti-biofouling, anti-fogging, antiicing, and anti-bacterial [7, 8]. The form of superhydrophobic surface control by roughness, and synthesis methods require can be divided into two categories: (i) modification the surface with low surface energy material (ii) making the surface rough with low surface energy used some technique $[9,10]$. Hierarchical structures play an important role in wetting property because of the area fraction of solid surface contact with liquid is much smaller than that of single-level structure, to explainable why hierarchical structures can generate superhydrophobic state, we depend on the wellknown theories, such as Wenzel and Cassie-Baxter models for the liquid drop on rough solid surfaces [11]. Variety materials used for fabrication the superhydrophobic surfaces including both the organic and inorganic materials [12]. Inorganic materials that do not contain carbon, and not deriving from living matter, such as $\mathrm{ZnO}$ and $\mathrm{TiO}_{2}$ have been of particular interest in recent years because of their potential practical applications, especially in decomposition of organic material from their surface under exposure to the UVillumination, therefore this material used as a non-wetting and self-cleaning surface, and also require high cost, multiple steps, special equipment and the critical conditions [13]. Organic materials such as polymeric materials that contain carbon or C-H bonds generally inherently hydrophobic, fabrication of surface roughness is the primary focus, the 
primary difference between inorganic compounds and organic compounds, that the organic compounds always contain carbon while most of the inorganic compounds do not contain carbon, also, almost all organic compounds contain C-H bonds or carbon-hydrogen [12]. Polymers (macro-molecules) form a very important class of materials they are all around us in everyday use in rubber, plastic, resins, adhesives and tapes, the word polymer derived from the Greek words, poly (many) and mer (parts or units) of high molecular mass each molecule of which consist of a very large number of the single structural units joined together in a regular manner [14]. The distinct characteristics polymer are: very resistant to chemicals, both thermal and electrical insulators, very light in weight with significant degrees of strength, processed in various ways, usually made of petroleum, but not always, used to make items that have no alternatives from other materials and polymers can made into clear, waterproof surface [15]. Polymers have been classified into three types; Natural polymer such as (natural silk, rubber, proteins, cellulose, starch, etc..); Semi synthetic polymer such as (hydrogenated, natural rubber, cellulosic, cellulose nitrate, methyl cellulose, etc..); Synthetic polymer which synthesized in the laboratory such (polyethylene, polyvinyl alcohol, polysulfone, polystyrene, polycarbonate, polypropylene, polyacrylonitrile, poly methyl methacrylate, etc..) [16]. Polycarbonates (PCs) are an important thermoplastic polymer which have organic functional groups linked together by carbonate groups (-O-CO-O-), PCs temperature resistant, non-toxic and exhibits excellent mechanical, optical and low water absorption properties [17]. Therefore, PCs are very attractive material using in a wide range of applications includes optical data storage devices (CDs and DVDs), bulletproof windows, food packaging, mineral water bottles, packaging, medical, machine parts, electronic equipments, building and self-cleaning [18, 19]. Moreover, they dissolve easily in organic solvents like (toluene, dichloromethane, formamide (DMF), tetrahydrofuran) [20]. Although these properties, PC surface exhibits low water CA $\left(\sim 84^{\circ}\right)$ [21]. Therefore, suffers from hydrophilic effect or partial wetting when contact with aqueous solutions, which severely limits of using PC in various applications of water repellency [22]. Various approaches devloped to transform a hydrophilic PC into superhydrophobic material such as ion beam irradiation, chemical treatments, plasma treatment [23, 24]. In fact, most of these methods which used to fabricate superhydrophobic surfaces are either using special apparatus or complicated and destabilized of synthesized surface after overtime in humid environments, also require multiple steps, specific substrates. Thus, it becomes urgent demand of preparing the superhydrophobic PC surfaces by inexpensive and simple method, and it will further greatly advanced the many applications. In contrast, rapid, simple, and low-cost phaseseparation method was applied to polymer materials in order to fabricate rough superhydrophobic surfaces [10]. In compared with other studies [25-28], this study, developed a novel approuch and low-cost technique for fabricating a hierarchical PC structure with a micro-nano-binary formation in one step, without the need for special instruments and low surface energy material modification by using solvent/nonsolvent selected and phase separation with different percentages of solvents.

\section{Methodology}

In this work superhydrophobicity surfaces synthesized using cast drop and phase separation technique, which is simple, low cost, and highly efficient method for Preparation hierarchical PC surface.

\section{Experimental}

\subsection{Materials}

PC resin was acquired from Sabic Saudia. Acetone $\left(\mathrm{C}_{3} \mathrm{H}_{6} \mathrm{O}\right.$; purity of $\left.99 \%\right)$ from Himedi India and absolute ethanol $\left(\mathrm{C}_{2} \mathrm{H}_{5} \mathrm{OH}\right)$ from the Netherlands were used as received. DMF $\left(\mathrm{C}_{3} \mathrm{H}_{7} \mathrm{NO}\right.$; purity of $\left.99.9 \%\right)$ was obtained from Scharlau Spain.

\subsection{Preparation of Superhydrophobic Surface}

The glass substrate was cleaned with ethanol, and deionized water for several times to remove the residuals and impurities from the surface of substrate. Resin of PC was dissolved at $55 \%$ and $65 \%$ acetone/DMF mixture solvents percentage, with ultrasonically dispersed for $30 \mathrm{~min}$, and magnetically stirred for $8 \mathrm{~h}$. After homogeneity, the solution was drop cast on a substrate using a micropipette (germany pipette), and evaporated at room temperature.

\subsection{Characterization}

The surface morphology of the Specimens was observed with a field emission scanning electron microscope (FESEM; Hitachi-S4160, Japan). The behavior of superhydrophobic and water CA of the surface was characterized on a homemade system at ambient temperature and this process was monitored by a high-speed CCD camera with a setting of 25 frames per second.

\section{Results and Discussion}

We fabricated PC superhydrophobic surfaces using phase separation method described above at room temperature with different percentage of acetone/ DMF mixture solvents. The surface structure is one of the key factors which need to control for formation superhydrophobic surface, also should have roughness on nano/micro-scale and should be hierarchical structure. Figure (1) shows the FESEM images of the PC superhydrophobic surfaces after adding $55 \%$ of the acetone/DMF mixture with water CAs of $153 \pm 2^{\circ}$. From the FESEM image, results showed the PC surface formed after treatment with mixture of solvent was rough and entirely composed of stacked particles. 


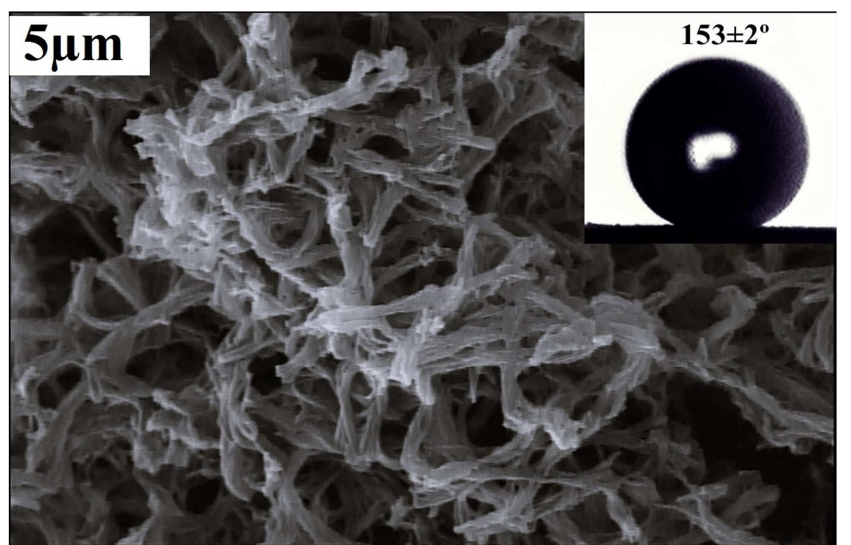

Figure 1. FESEM image of PC surface morphology after treatment with $55 \%$ acetone/DMF solvent mixture. Inset is $C A$.

Figures (2) show the FESEM image of the PC superhydrophobic surfaces after adding $65 \%$ of the acetone/DMF mixture with water CA of $156 \pm 2^{\circ}$. From this figures, FESEM image exhibited that the PC surface composed of flower-like shaped linked together centrally with branches offered roughness on the surface with a hierarchical micro/nano-binary formation. In order to discussion the effect of acetone/ DMF mixture solvents on formation of PC superhydrophobic surfaces, the difference in solubility and melting point played an important role in the formation of the hierarchical structure of the surface. According to the phase seperation method, acetone is a nonsolvent of PC resin that acts as a precipitator, and some aggregates appear with the addition of a non-solvent, and DMF is solvent to PC resin, Therefore, when the solvent mixture increased more polymer aggregates formed and caused hierarchical structure formation.

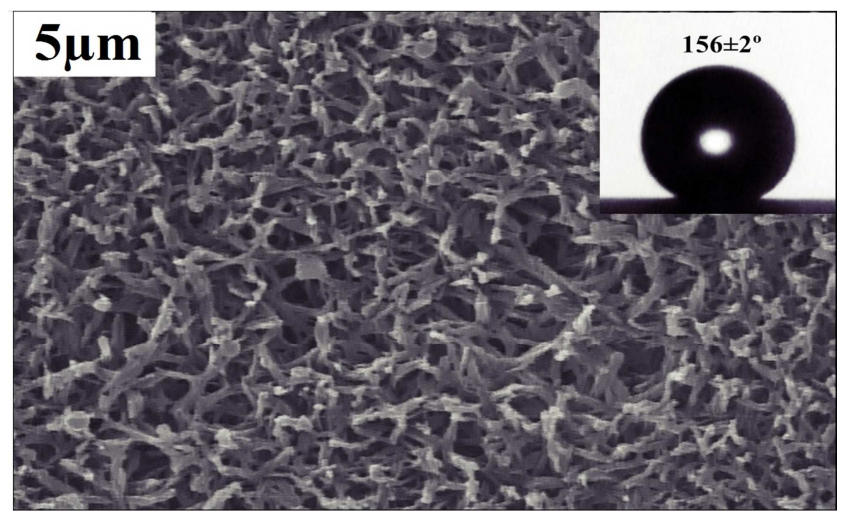

Figure 2. FESEM image of PC surface morphology after treatment with $65 \%$ acetone/DMF solvent mixture. Inset is $C A$.

The PC superhydrophobic surface was immersed in water with the temperatures ranging from $5^{\circ} \mathrm{C}$ to $45^{\circ} \mathrm{C}$ for $1 \mathrm{~h}$ to 155 days, the water $\mathrm{CA}$ remained higher than $150^{\circ}$, in order to evaluate.

\section{Conclusions}

A stable superhydrophobic PC surface has been obtained directly without addition of low-surface-energy compounds by a new simple approach. The water CAof the prepared superhydrophobic PC surface were $156 \pm 2^{\circ}$. Compared with other methods of synthetic superhydrophobic surfaces, the present method is very simpler to control, without adding any complicated process. It can also be easily scaled up to create large-area superhydrophobic surface structures and can be applied in the commercial manufacturing.

\section{Acknowledgements}

This author is grateful for the financial support received for this work from (Department of physics/ college of science / Al-Mustansiriya university / Baghdad / Iraq).

\section{References}

[1] Bharat Bhushan and Yong Chae Jung, "Natural and Biomimetic Arti-ficial Surfaces for Superhydrophobicity, SelfCleaning, Low Adhesion, and Drag Reduction", Progress in Mate- rials Science, Vol. 56, No. 1, pp. 1-108, (2011).

[2] Thomas Wagner, Christoph Neinhuis and Wilhelm Barthlott, "Wettability and Contaminability of Insect Wings as a Function of Their Surface Sculptures", Acta Zoologica, Vol. 77, No. 3, pp. 213-225, (1996).

[3] Michael Nosonovsky, "Superhydrophobic Surfaces and Emerging Applications: Non-Adhesion, Energy, Green Engineering", Current Opinion in Colloid \& Interface Science, Vol. 14, No. 4, pp. 270-280, (2009).

[4] Chih-Feng Wang, Wei-Yan Chen, Huy-Zu Cheng, and Shen-Li $\mathrm{Fu}$, "Pressure-proof superhydrophobic films from flexible carbon nanotube/polymer coatings", J. Phys. Chem. C, 114 (37), pp. 15607-15611 (2010).

[5] M. N. Valipour, F. C. Birjandi, J. Sargolzaei, "Super-nonwettable surfaces: a review", Colloid Surface. Vol. 448, 20, pp. 93-106, (2014).

[6] Liang liang Cao, Tyler P. Price, Michael Weiss, and Di Gao, "Super water-and oil-repellent surfaces on intrinsically hydrophilic and oleophilic porous silicon films", Langmuir, 24 (5), pp 1640-1643, (2008).

[7] Sumit Barthwal, Young Su Kim, Si-Hyung Lim, "Superhydrophobic and superoleophobic copper plate fabrication using alkaline solution assisted surface oxidation methods", Int. J. Precis. Eng. Manuf. 13, Vol. 13, Issue 8, pp. 1311-1315, (2012).

[8] Reiner Fürstner and Wilhelm Barthlott, "Wetting and selfcleaning properties of artificial superhydrophobic surfaces", Langmuir, pp. 956-961, (2005).

[9] E Elena Celia, Thierry Darmanin, Elisabeth Taffin de Givenchy, Sonia Amigoni, Frédéric Guittard, "Recent advances in designing superhydrophobic surfaces", J. Colloid Interface Sci, Vol. 402, pp. 1-18, (2013).

[10] H. Y. Erbil, A. L. Demirel, Y. Avc1, O. Mert, "Transformation of a simple plastic into a superhydrophobic surface", Science, Vol. 299, Issue 5611, pp. 1377-1380, (2003). 
[11] Donghui Chu, Akihiko Nemoto and Hiroshi Ito, "Biomimetic superhydrophobic polymer surfaces by replication of hierarchical structures fabricated using precision tooling machine and anodized aluminum oxidation", Microsyst Technol, Vol. 21, Issue 1, pp. 123-130, (2015).

[12] Adel M. A. Mohamed, Aboubakr M. Abdullah and Nathalie A. Younan, "Corrosion behavior of superhydrophobic surfaces: A review", Arabian Journal of Chemistry, Vol. 8, Issue 6, pp. 749-765, (2015).

[13] Zhang Xintong Zhang, Ming Jin, Zhaoyue Liu, Shunsuke Nishimoto, Hidenori Saito, Taketoshi Murakami, and Akira Fujishima, "Preparation and Photocatalytic Wettability Conversion of TiO2-Based Superhydrophobic Surfaces", Langmuir, pp. 9477-9479, (2006).

[14] Gowariker, V. R., Viswanathan, N. V. and Shreedhar, "Polymer Science", New Age International, ISBN : 978-81224-3813-0, (2015).

[15] Georg H. Michler and F J Baltá-Calleja, "Nano- and Micromechanics of Polymers Structure Modification and Improvement of Properties", Carl Hanser Verlag, ISBN: 9783-446-42767-9 (2012).

[16] Manas Chanda, "Introduction to Polymer Science and Chemistry", CRC Press, Taylor and Francis Group, ISBN 9781466553842, (2006).

[17] Marcia C Delpech, Fernanda M. B Coutinho and Maria Eunice S Habibe, "Bisphenol A-based polycarbonates: characterization of commercial samples", Polymer Test, Vol. 21, Issue 2, pp. 155-161, (2002).

[18] Chiu, S. T., Chen, S. H., Tsai, C. T., "Effect of metal chlorides on thermal degradation of (waste) polycarbonate", Waste Manag, pp. 252-259, (2006).

[19] Balart, R., Sanchez, L., Lopez, L., Jimenez, A., "Kinetic analysis of thermal degradation of recycled polycarbonate/acrylonitrilebutadiene-styrene mixtures from waste electric and electronic equipment", Polymer Degradation and Stability, Vol. 91, Issue 3, pp. 527-534, (2006).
[20] Schulz, U, "Review of modern techniques to generate anti reflective properties of thermoplastic polymers", Applied Optics, Vol. 45, Issue 7, pp. 1608-1618, (2006).

[21] Zhang X, Tan S, Zhao N, Guo X, Zhang X, Zhang Y, Xu J, "Evaporation of Sessile Water Droplets on Superhydrophobic Natural Lotus and Biomimetic Polymer Surfaces", Chem Phys Chem, pp. 2067-2070, (2006).

[22] Ning Zhao, Jian Xu, Qiongdan Xie, Lihui Weng, Xinglin Guo, Xiaoli Zhang, Lianghe Shi, "Fabrication of Biomimetic Superhydrophobic Coating with a Micro-Nano-Binary Structure", Macromolecular Rapid Communications, Vol. 26, Issue 13, pp. 1075-1080, (2005).

[23] Ning Zhao, Lihui Weng Dr., Xiaoyan Zhang, Qiongdan Xie, Xiaoli Zhang, Jian Xu Prof, "A Lotus-Leaf-Like Superhydrophobic Surface Prepared by Solvent-Induced Crystallization", ChemPhysChem, Vol. 7, Issue 4, pp. 824 827, (2006).

[24] Chaowei Guo Dr., Lin Feng Dr., Jin Zhai Dr., Guojie Wang Dr., Yanlin Song Prof., Lei Jiang Prof., Daoben Zhu Prof., "Large-Area Fabrication of a Nanostructure-Induced Hydrophobic Surface from a Hydrophilic Polymer", ChemPhysChem, Vol. 5, Issue 5, pp. 750-753, (2004).

[25] Yuehua Cui, Adam T. Paxson, Katherine M. Smyth, Kripa K. Varanasi, "Hierarchical polymeric textures via solventinduced phase transformation: A single-step production of large-area superhydrophobic surfaces", Colloids and Surfaces A: Physicochem. Eng. Aspects, Vol. 394, pp. 8-13, (2012).

[26] P. Jankowski, D. Ogonczyk, A. Kosinski,W. Lisowski, P. Garstecki, "Hydrophobic modification of polycarbonate for reproducible and stable formation of biocompatible microparticles", Lab Chip, Vol. 11, pp. 748-752, (2011).

[27] Seungcheol Go, Moonhee Han, and Younghyun Ahn, "Formation of Nanoporous Polycarbonate Surfaces and Their Chemical Modification for Superhydrophobicity", BulletinKorean Chemical Society, Vol. 33, pp. 3899-3902, (2012).

[28] Sharad D. Bhagat, Mool C. Gupta, "Superhydrophobic microtextured polycarbonate surfaces", Surface and Coatings Technology, Vol. 270, pp. 117-122, (2015). 